\title{
Development and Validation of an Interactive Liner Design and Impedance Modeling Tool
}

\author{
Brian M. Howerton ${ }^{1}$, Michael G. Jones ${ }^{2}$ \\ NASA Langley Research Center, Hampton, VA 23681-2199 USA \\ and \\ James L. Buckley ${ }^{3}$ \\ ASRC Aerospace Corp, Cleveland, OH 44135-3127 USA
}

\begin{abstract}
The Interactive Liner Impedance Analysis and Design (ILIAD) tool is a LabVIEW-based software package used to design the composite surface impedance of a series of smalldiameter quarter-wavelength resonators incorporating variable depth and sharp bends. Such structures are useful for packaging broadband acoustic liners into constrained spaces for turbofan engine noise control applications. ILIAD's graphical user interface allows the acoustic channel geometry to be 'drawn' in the liner volume while the surface impedance and absorption coefficient calculations are updated in real-time. A one-dimensional transmission line model serves as the basis for the impedance calculation and can be applied to many liner configurations. Experimentally, tonal and broadband acoustic data were acquired in the NASA Langley Normal Incidence Tube over the frequency range of 500 to $3000 \mathrm{~Hz}$ at 120 and $140 \mathrm{~dB}$ SPL. Normalized impedance spectra were measured using the Two-Microphone Method for the various combinations of channel configurations. Comparisons between the computed and measured impedances show excellent agreement for broadband liners comprised of multiple, variable-depth channels. The software can be used to design arrays of resonators that can be packaged into complex geometries heretofore unsuitable for effective acoustic treatment.
\end{abstract}

\section{Introduction}

$\mathrm{T}$ The turbofan engine is the dominant contributor to the overall aircraft noise; therefore reduction of the noise it produces is critical for aircraft compliance with current and future noise regulations. Providing a large component of the overall engine noise, fan noise has traditionally been alleviated by a combination of passive liner treatments and nacelle modifications. ${ }^{1,2}$ Conventional, passive liners consist of a honeycomb core bonded between a porous face sheet and an impervious backing plate. Such a configuration produces an array of independent, onedimensional, tuned waveguides that behave as local-reacting absorbers. The acoustic absorption spectra of such structures are characterized by a single peak at the system resonance frequency and its odd harmonics with significantly reduced absorption at other frequencies. One way to increase bandwidth is with cascaded layers judiciously tuned to different resonance frequencies, but manufacturing complexities, weight and cost have limited their practical application. ${ }^{3}$ Another approach (also with design and fabrication complications) is to tune clusters of adjacent resonators to prescribed frequencies over bandwidths of interest. ${ }^{4}$ The continuing push towards lower thrust-specific fuel consumption implies increased by-pass ratios (BPR $\geq 6$ ) with greater fan diameters, fan chord lengths, and consequently, lower fan rotational speeds. Fan blade design and contouring, through the use of lean and sweep, have been employed to modify the acoustic characteristics of the engine. These trends lead to a further shift of the fan noise spectrum to lower frequencies, accompanied by increased broadband noise as a significant contributor to the aircraft's community noise impact. Airframe drag reduction considerations imply engine nacelles that are shorter relative to their diameter, thus limiting lined-duct-treatment-to-diameter ratio. This trend, along with reductions in nacelle wall thickness, inhibits liner effectiveness since a deeper liner would typically be required to

\footnotetext{
${ }^{1}$ Research Scientist, Structural Acoustics Branch, MS 463, Senior Member AIAA.

${ }^{2}$ Senior Research Scientist, Structural Acoustics Branch, MS 463, Associate Fellow AIAA.

${ }^{3}$ Mechanical Engineer, MS 500.
} 
mitigate lower frequency noise. Clearly, there exists a need for advanced liner technologies and tools to meet the acoustic challenges of the next generation of aircraft.

The focus of the current effort is on developing enabling tools to design and analyze passive acoustic liners for portions of an engine heretofore unused. Practical applications hinge upon suitable packaging of conventional resonator absorbers into the space available while accommodating absorption bandwidth requirements, especially at the lower frequencies of interest. ${ }^{5}$ The main obstacle to achieving low frequency performance is limited liner depth. Previous research has shown how high-aspect ratio slot resonators can be configured with bends to produce a liner where the effective acoustic depth is much greater than the physical depth. ${ }^{6}$ The acoustic performance of such configurations is predictable using a 1-D propagation model.

It is the purpose of this paper to describe the results of an experiment assessing the applicability of the 1-D acoustic propagation model to groups of quarter-wavelength resonators incorporating bends to efficiently package designs of variable depth. The goal is to predict the composite surface impedance of an array of unequal length resonator channels that might be configured similar to that shown in the sketch of Fig. 1. Complexities arise, however, in determining the necessary channel lengths required to produce a target impedance spectrum and how to package the bent channels into the available space.

The Interactive Liner Impedance Analysis and Design (ILIAD) software package was developed to provide a convenient method to rapidly design and analyze various resonator configurations in real time. The LabVIEW-based software has a graphical interface to let the user 'draw' the liner channels in the design space. The effect of new channels, or modifications to existing channels on the calculated impedance and absorption spectra, are automatically calculated and displayed. In this investigation, several model liner geometries with single-frequency or broadband absorption characteristics are tested in the NASA Langley Normal Incidence Tube (NIT). Experimentally measured surface impedances are compared to predictions derived from the ILIAD software's transmission-line, 1-D propagation model.

\section{Liner Design and Analysis Software}

\section{A. Development of the ILIAD software}

An initial impediment to utilizing bends to package resonant liners in confined or even irregularly shaped spaces was the difficulty in visualizing the spatial relationships between resonator channels and the design space. ILIAD was developed to provide a graphical means to plot the design space and, using the keyboard and mouse, draw in the liner resonator channels. Figure 2 shows a screenshot of the user interface where the upper white area displays the current liner geometry and design space. The code was written in LabVIEW using a state-machine architecture allowing for straightforward implementation of new features. Simple text files define the geometry of the design space while resonator channels are represented by an array of $\mathrm{X}$ and $\mathrm{Y}$ coordinates of their vertices relative to the design space origin. As the vertices are defined, the code calculates segment lengths (based on the channel centerline) and average channel wall separation (the average of the distance between the two matching pairs of vertices that define a channel segment). Coordinate values for individual vertices can be entered or adjusted manually through numeric controls to precise locations, if required. Special functions allow the user to duplicate or spatially shift an existing channel or rotate a channel horizontally about its first vertex. Once a liner design is completed, it can be saved to a file for later recall. The resulting predicted normalized impedance spectra can be exported from the code directly into other analysis software or spreadsheets.

A key feature of ILIAD is the incorporation of a 1-D acoustic propagation prediction code to calculate impedance and absorption spectra in real time based on the current liner geometry. Thus, the user can instantly see the effect of alterations to the channel configuration. The impedance calculation is coded as a separate subroutine to allow for future modeling improvements with minimal changes to the main program. ILIAD automatically generates the input deck for the impedance calculation and runs that subroutine whenever the liner geometry is modified.

\section{B. The Multi-Channel Impedance Calculation (MCIC) prediction code}

The MCIC prediction code was developed as a general tool to calculate surface impedance spectra for various liner configurations based on liner geometry and material properties. Liner geometries can be as simple as a single resonant channel but the code can evaluate arrays of channels, the effects of variable depth, as well as the addition of facesheets and embedded septa. Thus, liners with single or multiple degrees of freedom can be analyzed. Integrating the impedance model as a separate subroutine within ILIAD allows for easy incorporation of updated models without significant modification to the main code.

The surface impedance spectra of the individual chambers are combined to calculate the effective impedance of the liner sample. Computation of these values is achieved by first determining the acoustic admittance for each 
channel $\left(\beta_{c h}=1 / \zeta_{c h}\right)$. Individual channel admittances are calculated from a Zwikker-Kosten transmission line model described in previous work. ${ }^{4,6,7}$ The effective admittance at the liner surface can then be calculated by:

$$
\beta_{s}=\Omega \sum_{i=1}^{N_{c h}} \beta_{c h}
$$

where $N_{c h}$ represents the number of liner chambers and $\Omega$ is the open area ratio of the liner surface (porosity). The liner's uniform, effective, surface impedance is computed using the relation $\zeta_{s}=1 / \beta_{s}$.

The MCIC impedance prediction code uses a transfer function format to calculate the admittance of an individual channel, potentially consisting of multiple elements, as indicated in Fig. 3 which shows the labeling scheme used to define such a multi-element channel. Wave transmission through a dissipative channel can be described by a forward transmission matrix of the following form:

$$
\left(\begin{array}{c}
p\left(x_{n+1}\right) \\
u\left(x_{n+1}\right)
\end{array}\right)=\left(\begin{array}{ll}
T_{11} & T_{12} \\
T_{21} & T_{22}
\end{array}\right)\left(\begin{array}{l}
p\left(x_{n}\right) \\
u\left(x_{n}\right)
\end{array}\right)
$$

where $T_{11}=T_{22}=\cosh (k \Gamma L), T_{12}=\zeta_{c} \sinh (k \Gamma L)$, and $T_{21}=\zeta_{c}^{-1} \sinh (k \Gamma L)$ with $\Gamma$ as the propagation constant, $\zeta_{c}$ as the characteristic impedance, $k$ as the free-space wave number and $L$ as the channel depth. The terminating condition for each channel is assumed to be a rigid, impervious boundary expressed in matrix form as:

$$
\left(\begin{array}{l}
p\left(x_{0}\right) \\
u\left(x_{0}\right)
\end{array}\right)=\left(\begin{array}{l}
1 \\
0
\end{array}\right)
$$

The details of the model terms can be found in the works previously cited and in a companion paper on the development of the impedance model. ${ }^{8}$

\section{Experimental Approach}

\section{A. Liner Samples}

Four liner samples were fabricated for this investigation using additive manufacturing processes. The first liner sample was fabricated using conventional, resin-based stereolithography (SLA). A process known as Direct Metal Laser Sintering (DMLS) was employed to build the remaining three samples. Both SLA and DMLS allow the forming of solid, 3-D models by application of laser light to locally harden liquid resin (SLA) or fuse particles of powdered metal together (DMLS). A computer-controlled laser beam is scanned over the surface of a layer of the build media, tracing the area of one layer of a part causing that layer to fuse. Successive layers are built up until the entire part is formed. Part tolerances are to within +/- $0.05 \mathrm{~mm}$ (0.002 in) of the target dimensions. DMLS allows for the creation of blind chambers and passageways with smaller cross-sectional area than is possible with resin-based SLA since the unfused excess powder can be easily shaken out of the part. The viscosity of current stereolithography resins prevent gravity-assisted draining of small-diameter channels. Figure 4 shows a typical liner sample created using the DMLS process. Figure 5 is a cutaway of one of the samples detailing the internal resonator chambers.

The four liner samples are labeled C1 and S1-S3 and share certain physical characteristics. All samples have a square cross-section with a width of $63.5 \mathrm{~mm}$ ( $2.5 \mathrm{in}$.) and are of monolithic construction with an embedded array of circular (C1) or square (S1-S3) cross-section channels. They present an active area of $50.8 \mathrm{~mm} \times 50.8 \mathrm{~mm}$ (2 in x 2 in) to the incident plane waves in the NIT. The liner sample's active surface area matches the dimensions of the NIT's waveguide opening to provide an airtight seal for the test samples.

Liner C1 uses $1.83 \mathrm{~mm}$ (0.072 in) dia. channels while the square channels of S1-S3 have a width of $1.12 \mathrm{~mm}$ (0.044 in). Channel cross-section area is invariant (excluding bends) throughout the channel length. The channels are rigidly terminated within the structure itself and are intended to be acoustically isolated. The use of square crosssection channels, rather than round, for the metal samples resulted from a limitation of the DMLS process. Flat or slightly rounded surfaces formed perpendicular to the build direction over a cavity have a propensity to sag downward due to lack of support. Square channels rotated $90^{\circ}$, relative to the sample build direction, to form a 
diamond shape prevent this sagging in the horizontal resonator chambers. Liner C1 uses 168 channels per sample to achieve a percent-open-area (POA) of $17.1 \%$ while Liners S1-S3 employ 585 channels per sample (POA=28.3\%).

Liner $\mathrm{C} 1$ is the baseline case where the channels all have the same length of $42.88 \mathrm{~mm}$ (1.688 in.) corresponding to a resonant frequency of approximately $2 \mathrm{kHz}$. A photograph of this liner sample is shown in Figure 6 while Figures 7-8 show frontal and orthographic cutaways of the channel configuration. This liner sample is also used in a companion paper by Jones, et al. ${ }^{8}$ Liner S1 is similar to C1 in channel length but, as stated earlier, employs a greater number of smaller diameter channels. Figures 9-10 show frontal and orthographic cutaways of the liner channel configuration. Liner S2 employs groups of nine straight channels of various depths in an attempt to increase the absorption bandwidth across a frequency range of approximately 1400 to $3000 \mathrm{~Hz}$. Figures 11-12 show frontal and orthographic cutaways of this channel configuration. Figure 13 provides details of an individual channel group and the corresponding length of each channel. Liner S3 is a repackaging of the S2 channels to incorporate bends for the purpose of reducing the overall liner depth. Thus, while the total channel lengths for Liner S3 are the same as S2, the physical liner depth is reduced from $61.2 \mathrm{~mm}$ (2.41 in.) to $43.48 \mathrm{~mm}$ (1.712 in.). Figures 14-15 show frontal and orthographic cutaways of this channel configuration. Figure 16 provides details of an individual channel group and the corresponding length of each channel.

\section{B. Impedance Measurements}

Impedance spectra for each sample were obtained in the NIT via the Two-Microphone Method (TMM). ${ }^{9,10}$ A group of six electromagnetic acoustic drivers couple radially into a cylindrical tube that transitions to the $50.8 \mathrm{~mm} \times 50.8 \mathrm{~mm}$ ( 2 in $\times 2$ in) square cross-section waveguide. The key feature of the TMM is the efficient acquisition of the complex transfer function spectra between two, flush-mounted microphones strategically located in the standing wave field from which the complex reflection factor is calculated. Transfer function accuracy is assured by a microphone switching technique that avoids labor intensive amplitude and phase calibrations. Figure 17 shows the general arrangement of the NIT apparatus. For all tests, acoustic excitation was tonal from $500 \mathrm{to} 3000 \mathrm{~Hz}$ in $100 \mathrm{~Hz}$ increments. Data acquisition and analyses were performed in $100 \mathrm{~Hz}$ increments from 500 to $3000 \mathrm{~Hz}$ using a spectrum analyzer configured to a $12.5 \mathrm{~Hz}$ bin width. Data were acquired at sound pressure levels (SPL) of $120 \mathrm{~dB}$ and $140 \mathrm{~dB}$ as measured by a third, calibrated, reference microphone located a fraction of the shortest wavelength of interest $(6.35 \mathrm{~mm}, .025$ in) from the sample face. A computer controlled spectrum analyzer was employed in conjunction with custom software to acquire acoustic data and generate output files for analysis.

\section{Discussion of Results}

Figure 18 is a plot of the measured, normalized impedance spectra (relative to the characteristic impedance of air) for Liner C1 at 120 and $140 \mathrm{~dB}$. The near-perfect agreement between the two SPL's suggests a high degree of linearity. The same comparison is shown in Figure 19 for Liner S1 with similar results. This behavior was expected because the liner samples contain small diameter channels. This trend was observed in the measured data for all liner samples, so only results from the $140 \mathrm{~dB}$ cases are presented for the sake of brevity.

The use of square-shaped channels for the latter liner samples made it necessary to choose an appropriate channel diameter (D) for use with the MCIC code. For the prediction calculations, the value of D was based on the concept of equivalent diameter. This quantity is defined as the diameter of a circle having the same area as the channel cross-section. This value was $1.26 \mathrm{~mm}$ (0.0496 in) for the channel geometry of Liners S1-S3. Use of hydraulic diameter was also considered and analyzed. However, the resulting impedance comparisons with measured data for the S2 and S3 broadband designs, which are of the most interest, were not as favorable.

A comparison is provided in Figure 20 of the measured impedance measurements with the MCIC-calculated impedance spectra for Liner C1. The excellent agreement between the measured and calculated spectra implies that the model is adequately representing the absorption process. Figure 21 shows a similar comparison for the absorption coefficient where a slight shift in frequency and a small under-prediction of the magnitude is noted for the calculated absorption peak relative to the measured data.

When a similar comparison was made for Liner S1, there were significant deviations between the impedance spectra as shown in Figure 22. The normalized resistance was under-predicted across the entire frequency range with the deviation in $\theta$ growing with frequency. A similar trend was observed with the reactance spectra. A plot of the absorption coefficient spectum, shown in Figure 23, highlights the effect of these differences. The frequency of the measured absorption peak is approximately $250 \mathrm{~Hz}$ lower and significantly higher in magnitude relative to the predicted absorption spectra. In essence, the liner sample behaves as if the channels are longer than their physical length and with greater absorption efficiency than predicted. Possible explanations for this result will be presented later. 
Measured and predicted impedance spectra for the variable-depth Liner S2 are shown in Figure 24. The predictions track the measurements much more closely compared to the S1 sample indicating that the inclusion of variable depth improves the predicted impedance spectra. However, both the measured resistance and reactance curves are shifted to lower frequencies relative to the predicted values. The magnitude of this shift is not as pronounced as was observed for the S1 sample. Absorption coefficient spectra are shown in Figure 25. The high absorption coefficients above about $1200 \mathrm{~Hz}$ illustrate the intended broadband performance. It is noteworthy that this design, with only nine different channel lengths, provides substantial absorption $(\alpha \geq 0.5)$ over an $1800 \mathrm{~Hz}$ bandwidth. Predictions closely match the measured data for most of the frequency range with significant deviations only at the frequency extremes.

Similar results are achieved for Liner S3 (Figure 26) with slightly improved comparisons below $1200 \mathrm{~Hz}$ relative to S2. The frequency shift observed in the S1 and S2 samples is not as prominent in the S3 sample leading to a better match of the predicted absorption spectra to the measured data (Figure 27). Note that the absorption results for Liner S3 show equivalent performance with S2, even though the depth of S3 is nearly 30\% less than that of S2.

The comparison results (especially those for Liner S1) led to additional testing and numerical verification of the impedance model to rule out measurement and computational errors. No such errors were found. The increased measured resistance values may be attributable to the DMLS manufacturing process, as the surface finish of such parts cannot be categorized as 'smooth'. Roughness in the interior walls of the resonant channels would produce an effective reduction in channel diameter and, thus, present a greater acoustic resistance. Numerical studies suggest that reducing the channel diameter could raise the predicted resistance spectra sufficiently to improve comparisons with some of the measured data. However, there was no consistent correction that could be applied to the prediction calculations that would improve comparisons across all liner samples.

Further study is warranted to determine the cause of the discrepancy between the results from the MCIC model and the NIT data. Since the performance of the straight, constant-depth, round channel design of Liner C1 was predicted successfully, there could be some aspects of acoustic propagation through small-diameter square channels that is not captured in the current model. Alternatively, the DMLS process itself may be a contributing factor and could be isolated by testing geometrically identical parts made using Liner C1's resin-based process.

\section{Concluding Remarks}

The ILIAD software package has been developed to simplify the design and analysis of multi-channel, resonant absorbing liner systems. Previous studies have demonstrated that liners consisting of multiple, variable-depth chambers can be used to achieve broadband sound absorption. Coupled with a 1-D impedance propagation model, ILIAD can predict the composite normalized surface impedance of such liners. The ability to predict the performance of liners incorporating channels with variable depth and sharp bends opens the design space to consider liners for areas previously impractical to achieve meaningful noise reduction. The ability to rapidly modify the liner geometry and get immediate feedback on the acoustic performance of those modifications allows for the design of 'tailored' liners to target a desired absorption spectrum. Comparisons of the resulting predictions with experimental data from the NASA Langley Normal Incidence Tube showed excellent agreement over the frequency range from $500 \mathrm{~Hz}$ to $3000 \mathrm{~Hz}$ for these broadband liner samples.

Two constant-depth liner samples were also considered. The first incorporated circular channels, and was fabricated using conventional, resin-based stereolithography. The current model accurately predicted the surface impedance of this sample. The second sample incorporated square channels, and was fabricated using Direct Metal Laser Sintering. Comparisons between the predicted and measured impedance spectra were not nearly as good for this sample. Instead, the measured data for the latter sample suggest the channel depths are deeper than the physical dimensions. This intriguing result suggests the possibility of creating future liner designs to achieve improved absorption at lower frequencies than generally assumed possible. Clearly, further research regarding the effects of channel shape and manufacturing process should be considered.

The choice of an equivalent diameter for rectangular channels has a marked effect on the calculated impedance spectra and it is not entirely clear which definition should be used. Additional study is warranted and will be a topic of future work.

\section{Acknowledgements}

The authors would like to express appreciation to Carol Harrison, Robert Andrews and Michael Powers of NASA Langley Research Center along with Christopher Hughes of NASA Glenn Research Center for their contributions to this study. This work was funded by the Subsonic Fixed Wing Project of NASA's Fundamental 
Aeronautics Program and the Environmentally Responsible Aviation Project of NASA's Integrated Systems Research Program.

\section{References}

1،"The NASA acoustically treated nacelle program," Proceedings of the Aircraft Noise Symposium (Acoustical Duct Treatments for Aircraft), Invited Tutorial Papers presented at the $77^{\text {th }}$ Meeting of the Acoustical Society of America, 8, Apr. 1969, Philadelphia, PA.

2“Study and Development of Turbofan Nacelle Modifications to Minimize Fan-Compressor Noise Radiation,” Volume IV Flight-worthy Nacelle Development, Prepared by The Boeing Company, NASA CR-1714, Jan. 1971.

${ }^{3}$ Bielak, G. W., Premo, J. W., and Hersh, A. S., “Advanced Turbofan Duct Liner Concepts,” NASA CR-1999-209002, 1999.

${ }^{4}$ Parrott, T.L., Jones, M. G., "Parallel-element liner impedances for improved absorption of broadband sound in ducts,” Noise Control Engineering Journal, Vol. 43, No. 6, Nov. 1995.

${ }^{5}$ U. S. Patent: Low Noise Fan Exit Guide Vane, 7,334,998.

${ }^{6}$ Howerton, B. M., Parrott, T. L., "Validation of an Acoustic Impedance Prediction Model for Skewed Resonators," AIAA 2009-3143.

${ }^{7}$ Zwikker, C. Kosten, C., “Sound Absorbing Materials,” Elsevier, Amsterdam, 1949.

${ }^{8}$ Jones, M. G., Howerton, B.M., Ayle, E., "Evaluation of Parallel-Element, Variable-Impedance Broadband Acoustic Liner Concepts,” $18^{\text {th }}$ AIAA/CEAS Aeroacoustics Conference, Colorado Springs, CO, June 2012 (submitted for publication).

"'Standard Test Method for Impedance and Absorption of Acoustical Materials using a Tube, Two Microphones, and a Digital Frequency Analysis System,” ASTM E1050-90, 1990.

${ }^{10}$ Jones, M. G., and Stiede, P. E., “Comparison of Methods for Determining Specific Acoustic Impedance,” Journal of the Acoustical Society of America, Vol. 101, No. 5, 1997, pp. 2694-2704. 


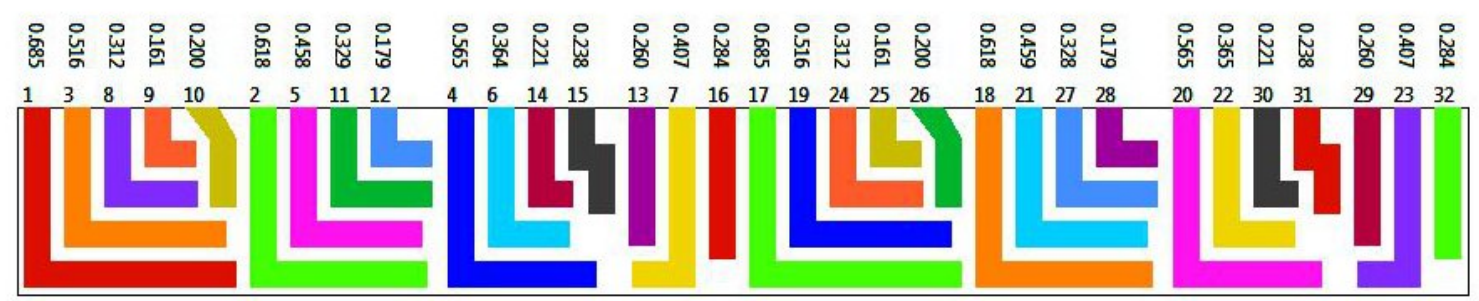

Figure 1. Sketch of notional liner using bent channel resonators to reduce physical liner depth. Numbering indicates the order it was added to the design (lower row) and total channel length in inches (upper row).

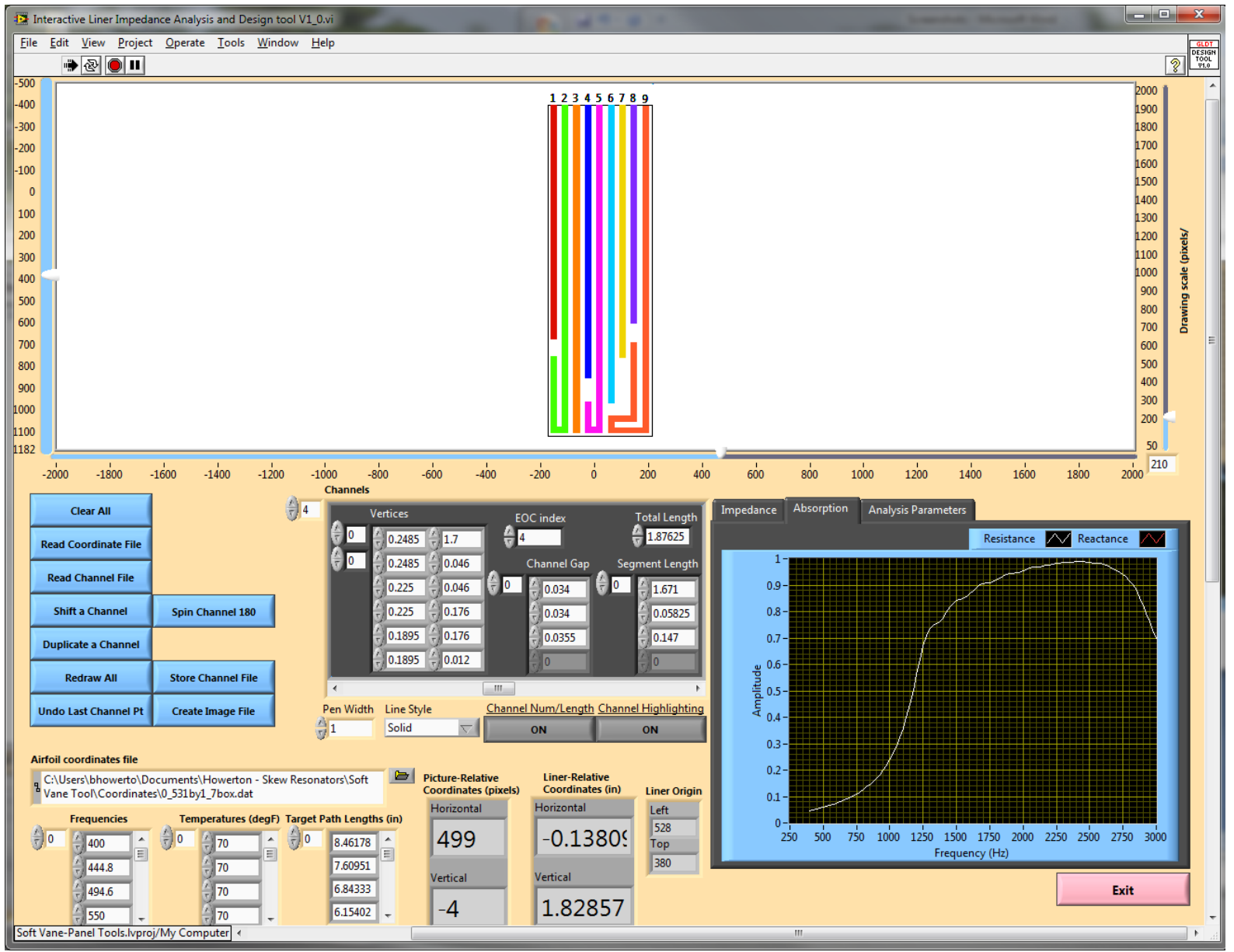

Figure 2. Screenshot of ILIAD software user interface during the design of Liner S3. 


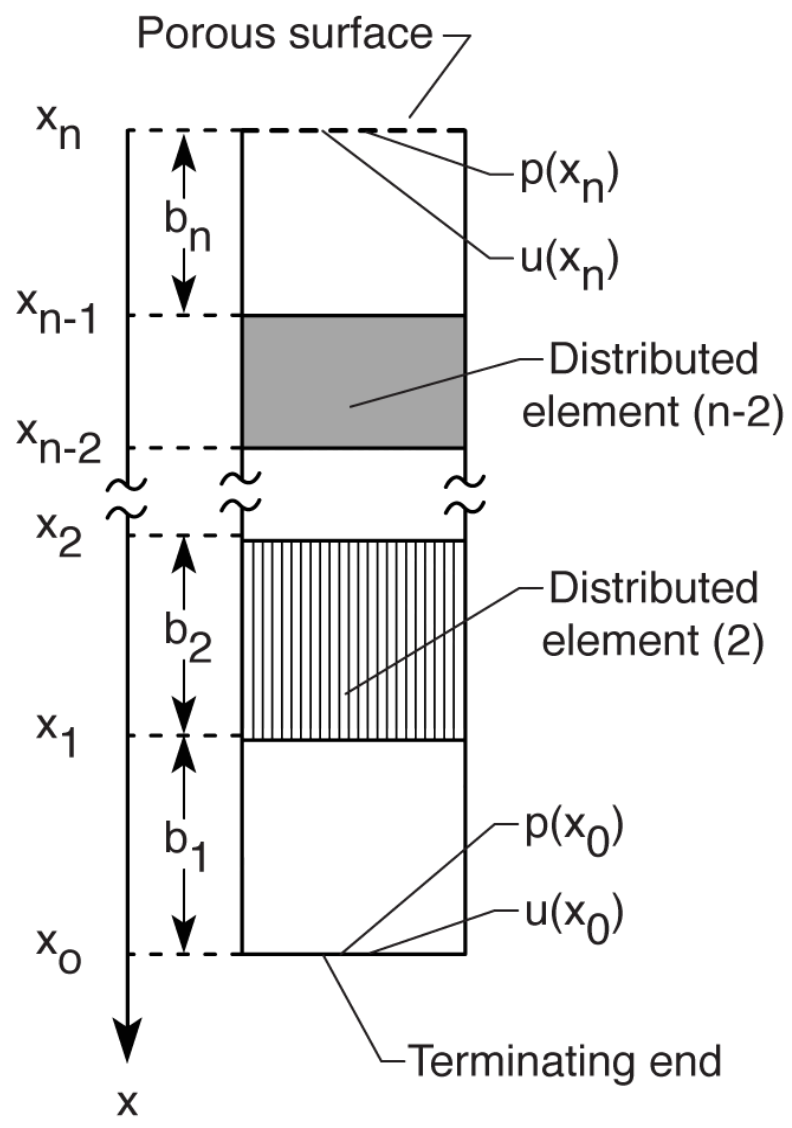

Figure 3. Labeling scheme for elements in a single, isolated channel of a multi-channel resonator system that is amenable for the 1-D Zwikker-Kosten waveguide propagation model. 


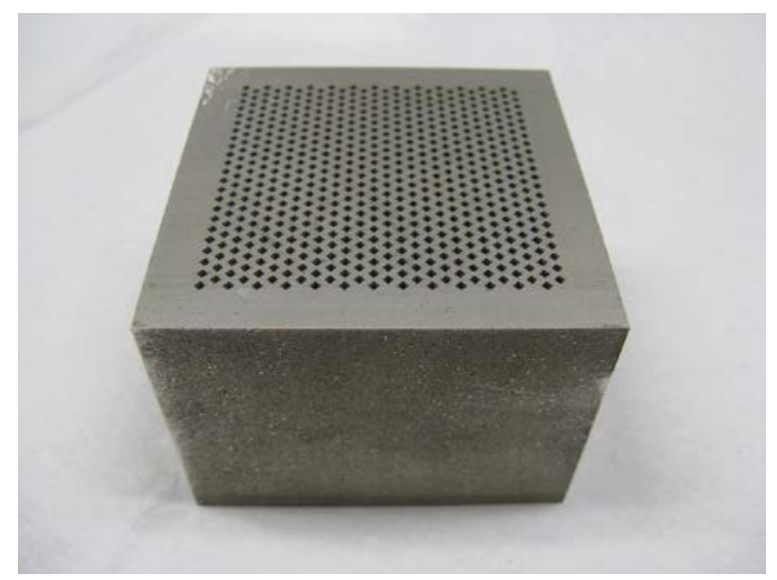

Figure 4. Direct Metal Laser Sintering (DMLS) liner sample.

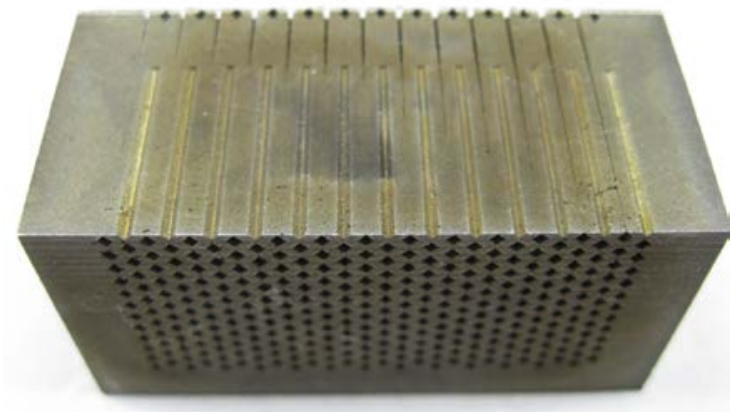

Figure 5. Cutaway of sample showing internal resonator detail.

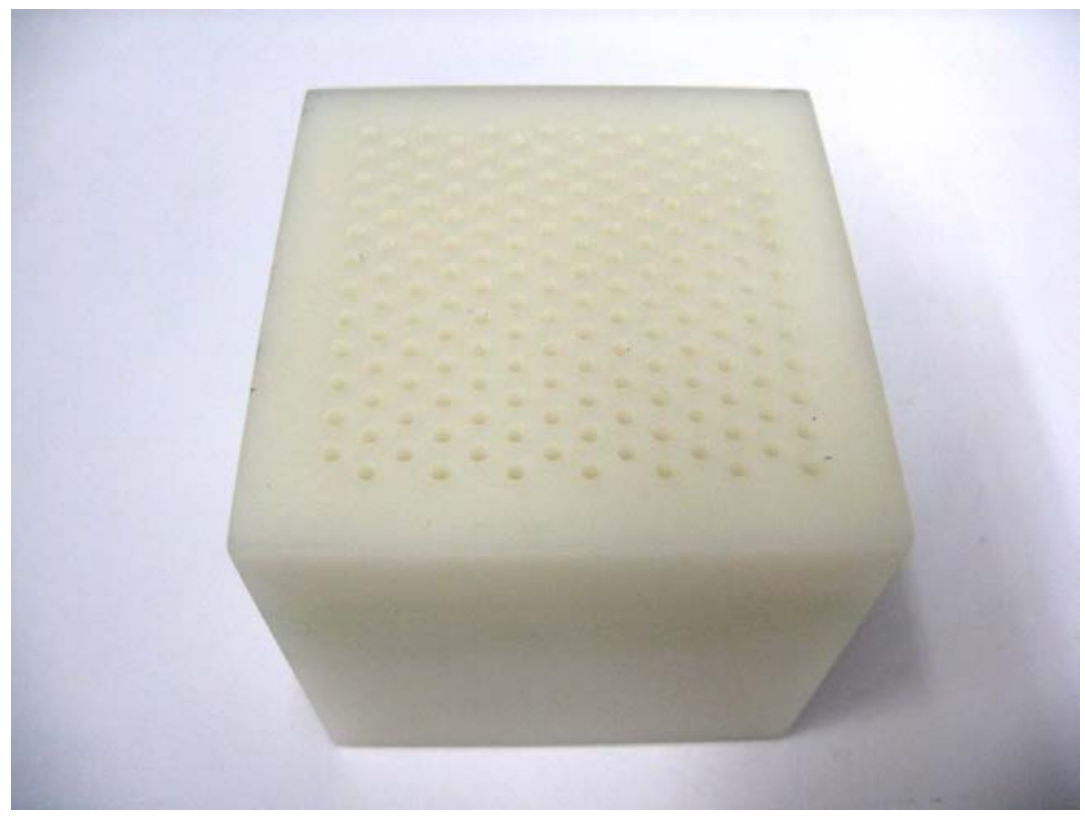

Figure 6. Liner C1 (constant depth, round, straight channels). $L=42.88 \mathrm{~mm}(1.668 \mathrm{in})$ 


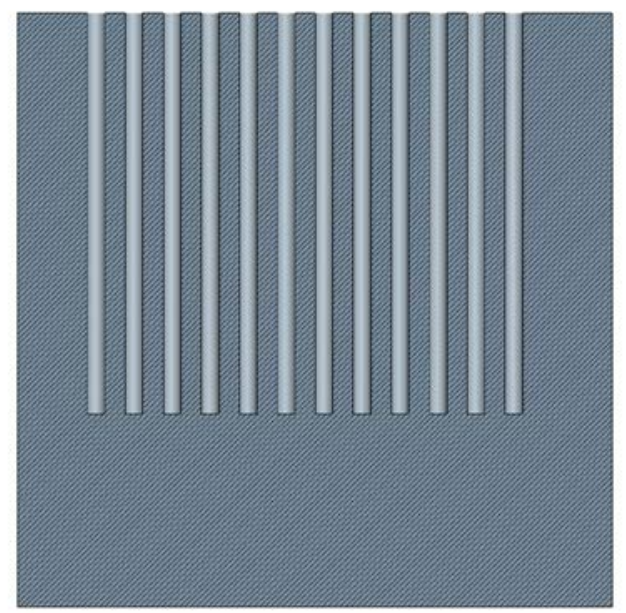

Figure 7. Liner C1 (constant depth, round, straight channels). $\mathrm{L}=\mathbf{4 2 . 8 8} \mathrm{mm}$ (1.668 in) - Frontal cutaway view.

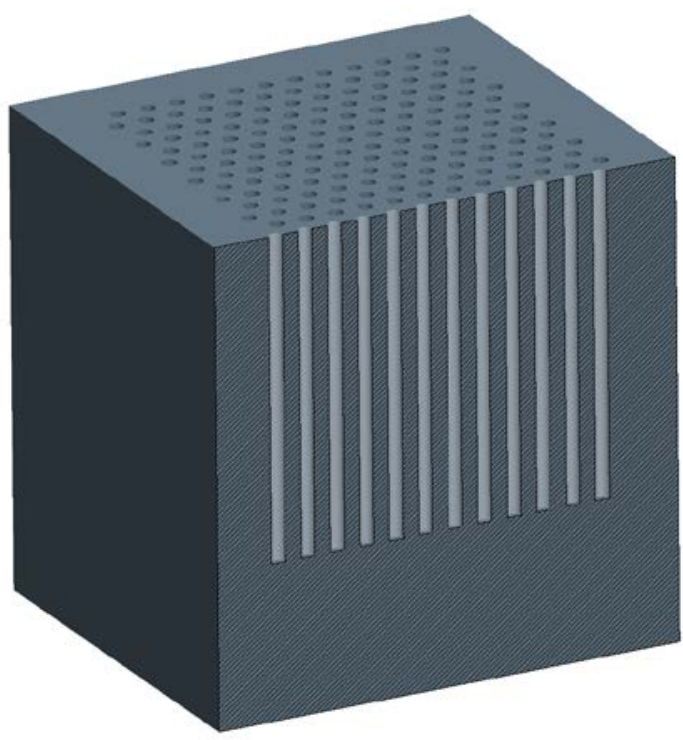

Figure 8. Liner C1 (constant depth, round, straight channels). $L=42.88 \mathrm{~mm}$ (1.688 in) - Orthographic cutaway view.
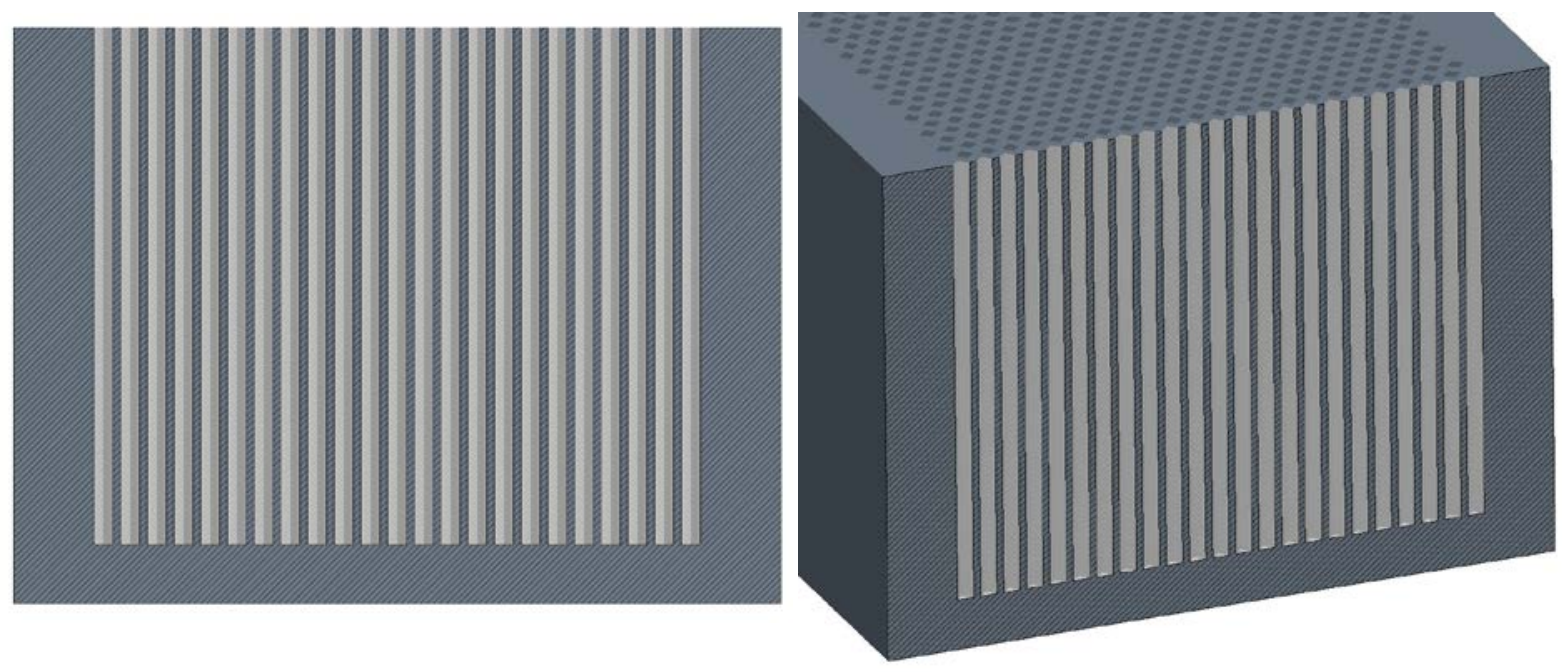

Figure 9. Liner S1 (baseline - constant depth,, square, straight channels). $L=42.88 \mathrm{~mm}$ (1.668in) Frontal cutaway view.
Figure 10. Liner S1 (baseline - constant depth, square, straight channels). $L=42.88 \mathrm{~mm}$ (1.688in) Orthographic cutaway view. 


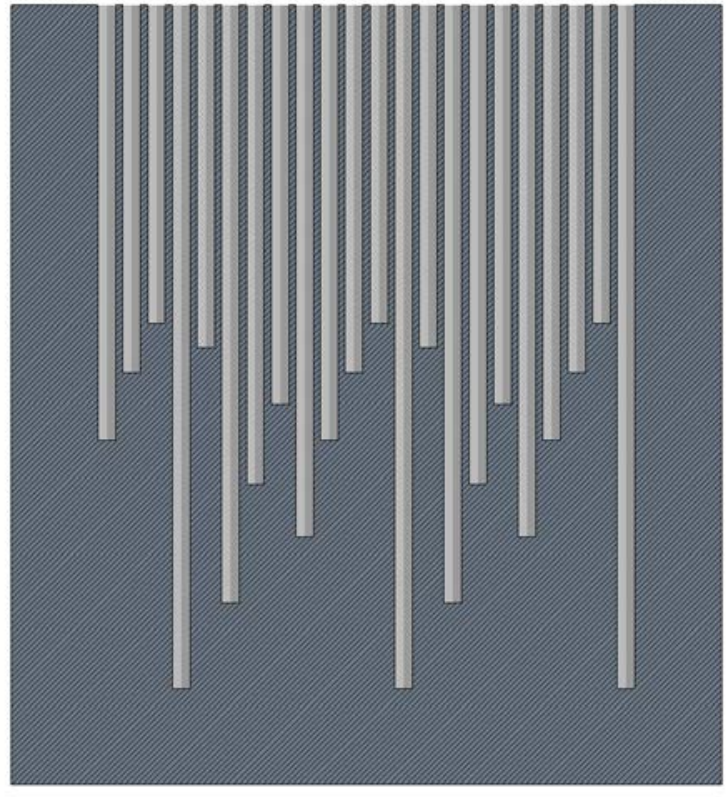

Figure 11. Liner S2 (variable depth, square, straight channels) - Frontal cutaway view.

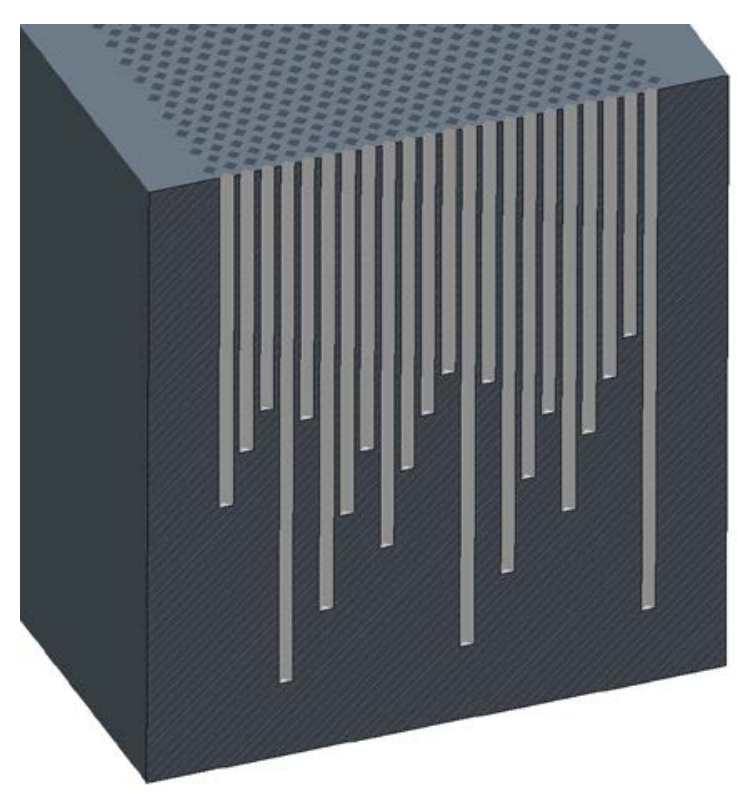

Figure 12. Liner S2 (variable depth, square, straight channels) - Orthographic cutaway view. 


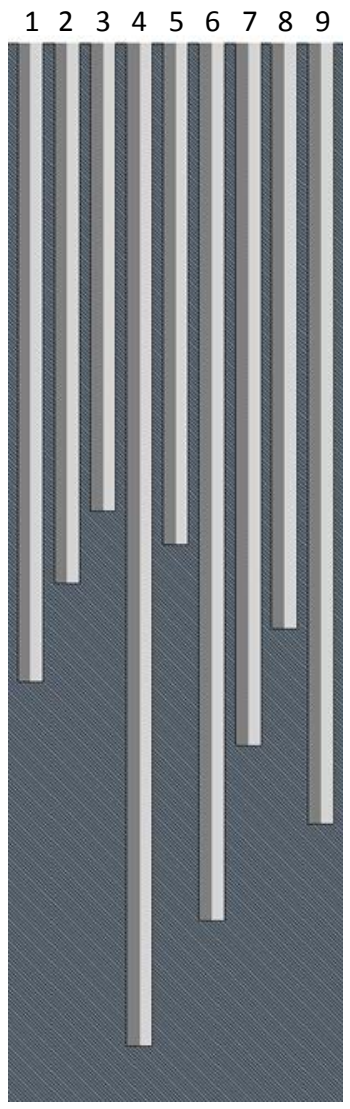

\begin{tabular}{|c|c|}
\hline Chamber & $\begin{array}{c}\text { Total Length } \\
\mathrm{mm} \text { (inches) }\end{array}$ \\
\hline 1 & $38.99(1.535)$ \\
\hline 2 & $32.97(1.298)$ \\
\hline 3 & $28.58(1.125)$ \\
\hline 4 & $61.26(2.412)$ \\
\hline 5 & $30.63(1.206)$ \\
\hline 6 & $53.59(2.110)$ \\
\hline 7 & $42.88(1.688)$ \\
\hline 8 & $35.74(1.407)$ \\
\hline 9 & $47.65(1.876)$ \\
\hline
\end{tabular}

Figure 13. Liner S2 channel group numbering and lengths. 

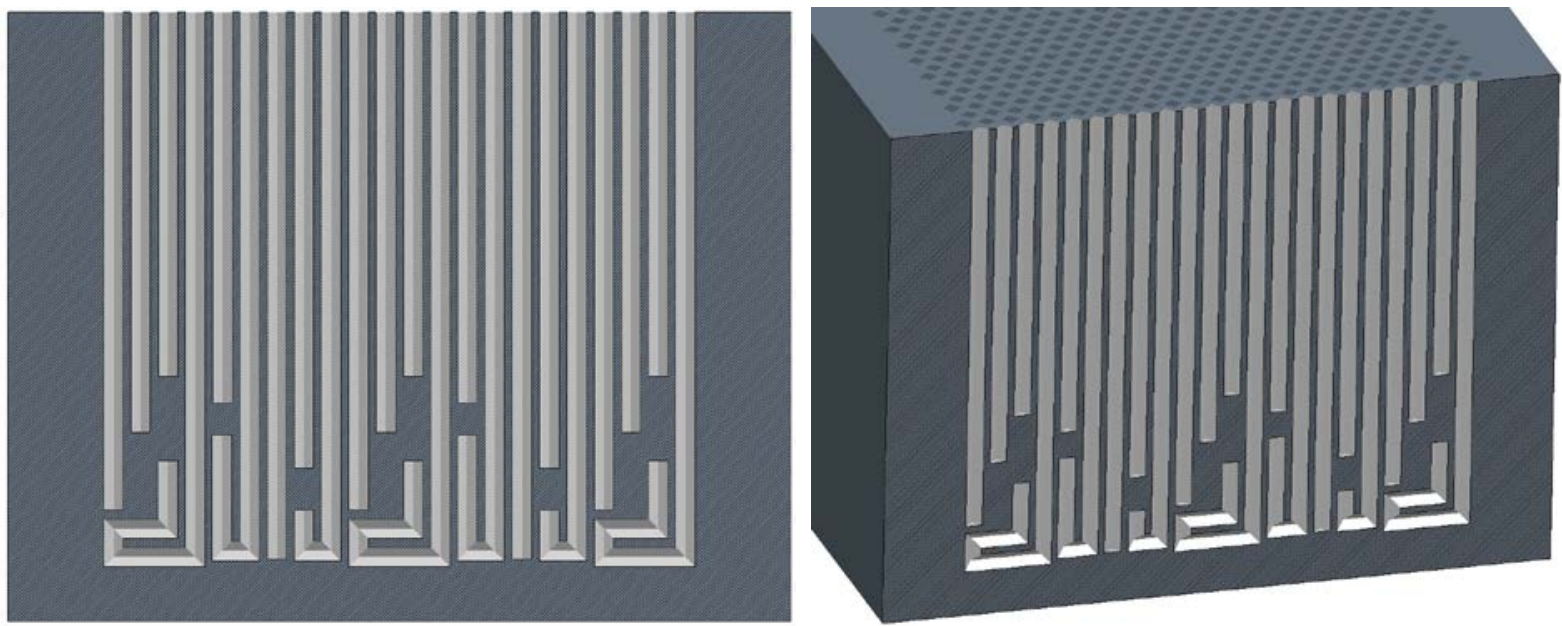

Figure 14. Liner S3 (variable depth, square, bent channels) - Frontal cutaway view.

Figure 15. Liner S3 (variable depth, square, bent channels) - Orthographic cutaway view.

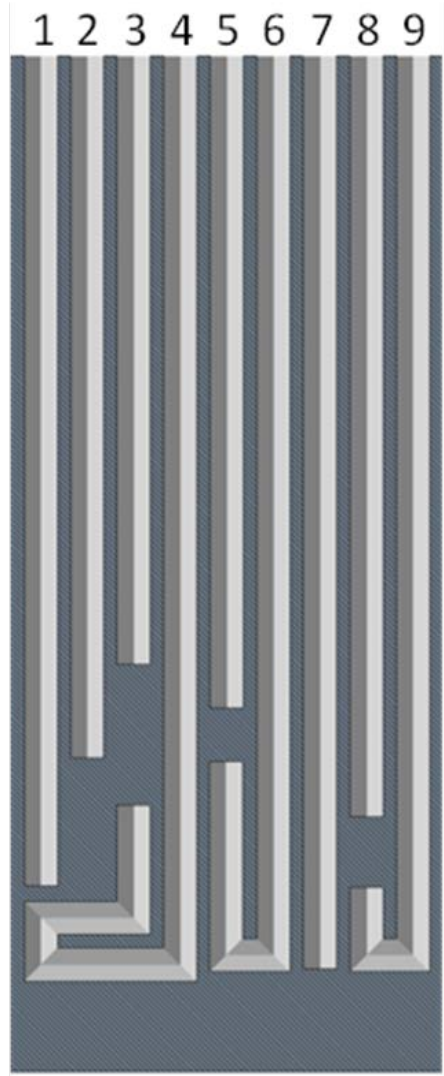

\begin{tabular}{|c|c|}
\hline Chamber & $\begin{array}{c}\text { Total Length } \\
\mathrm{mm} \text { (inches) }\end{array}$ \\
\hline 1 & $38.99(1.535)$ \\
\hline 2 & $32.97(1.298)$ \\
\hline 3 & $28.58(1.125)$ \\
\hline 4 & $61.26(2.412)$ \\
\hline 5 & $30.63(1.206)$ \\
\hline 6 & $53.59(2.110)$ \\
\hline 7 & $42.88(1.688)$ \\
\hline 8 & $35.74(1.407)$ \\
\hline 9 & $47.65(1.876)$ \\
\hline
\end{tabular}

Figure 16. Liner S3 channel group numbering and lengths. 


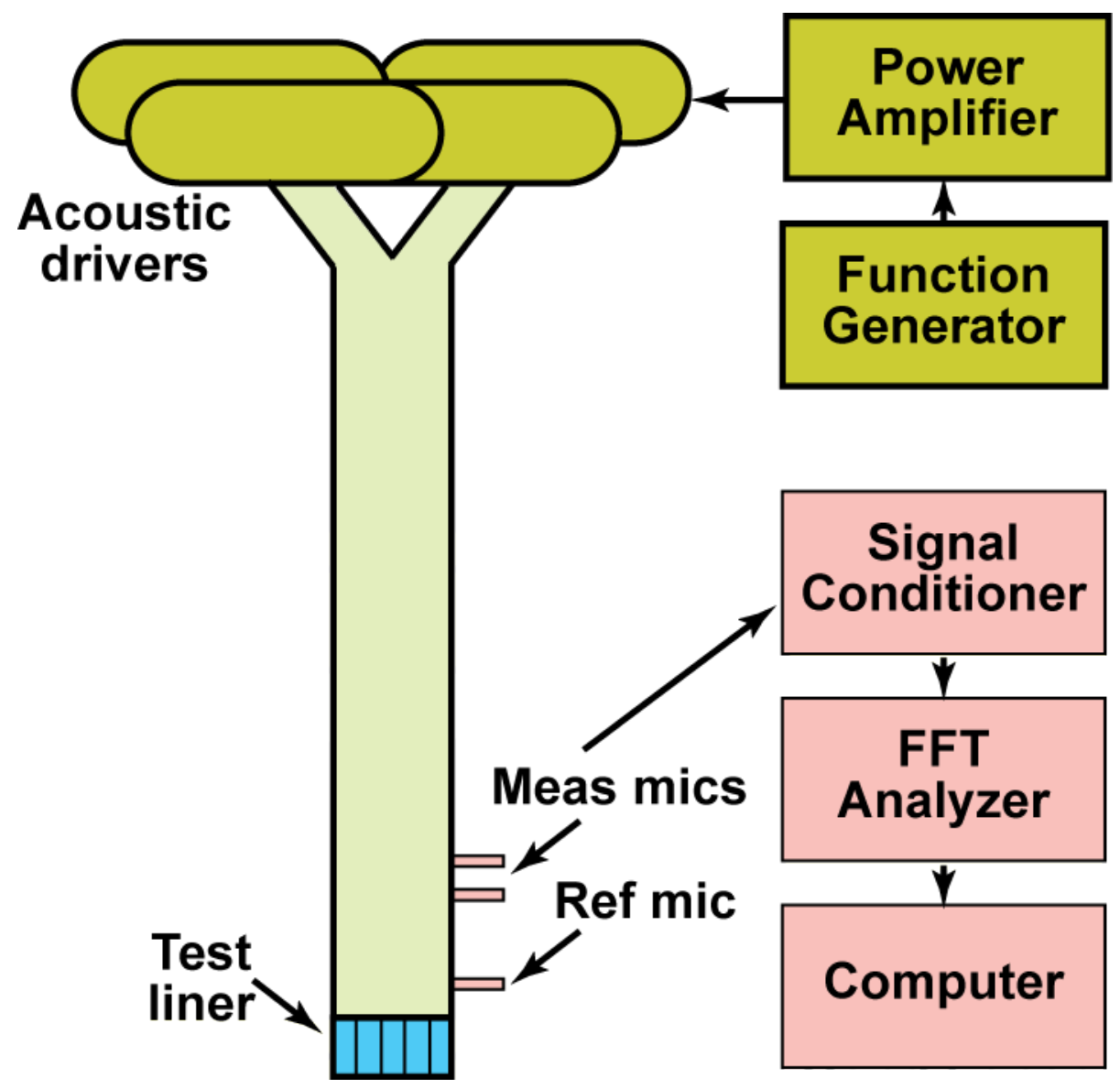

Figure 17. Sketch of Normal Incidence Tube (NIT) and associated instrumentation. 


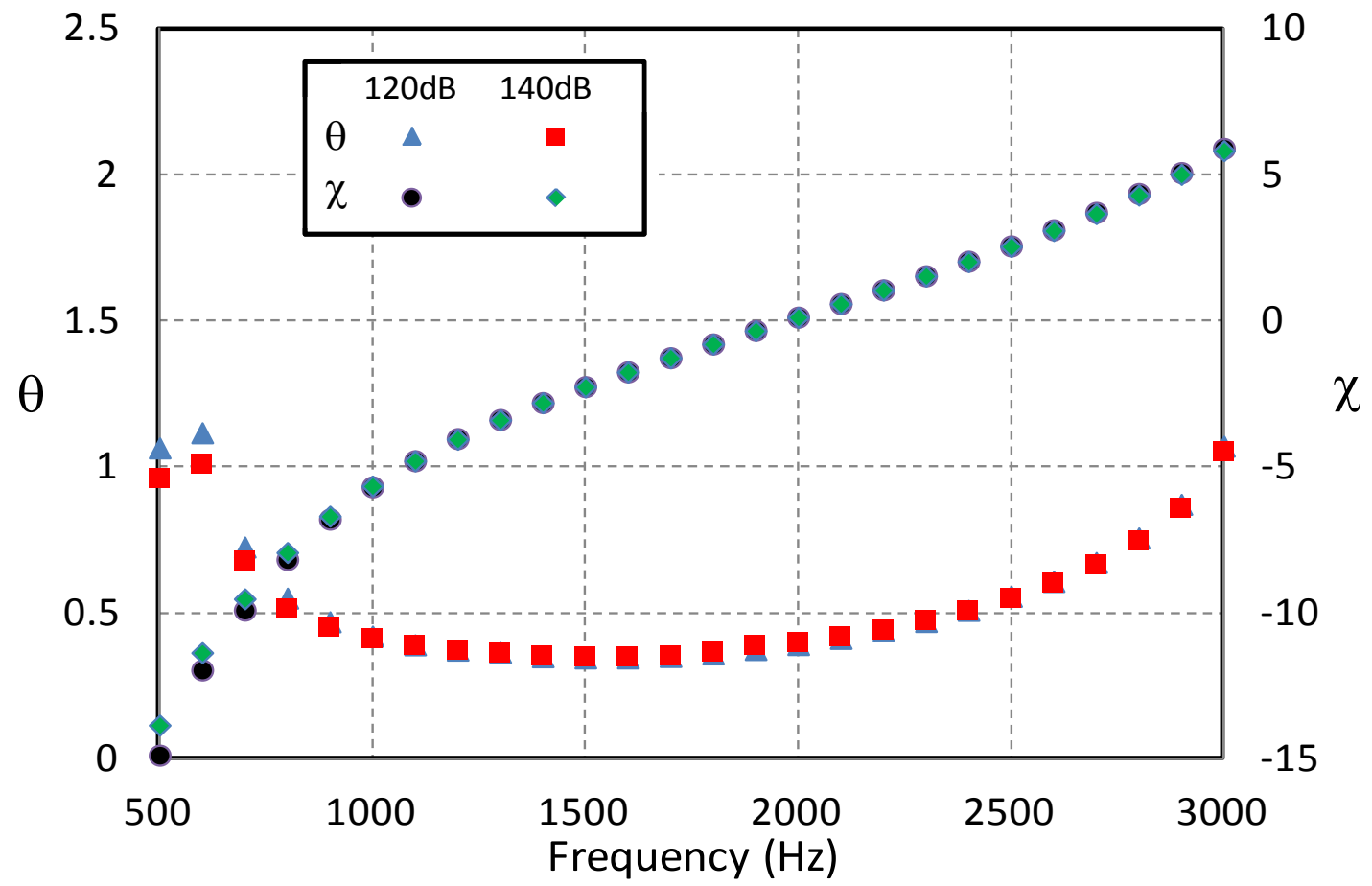

Figure 18. Measured normalized impedance $(\zeta=\theta+i \chi)$ spectra for Liner C1 (constant depth, round, straight channel), $S P L=120 \mathrm{~dB}$ and $140 \mathrm{~dB}$.

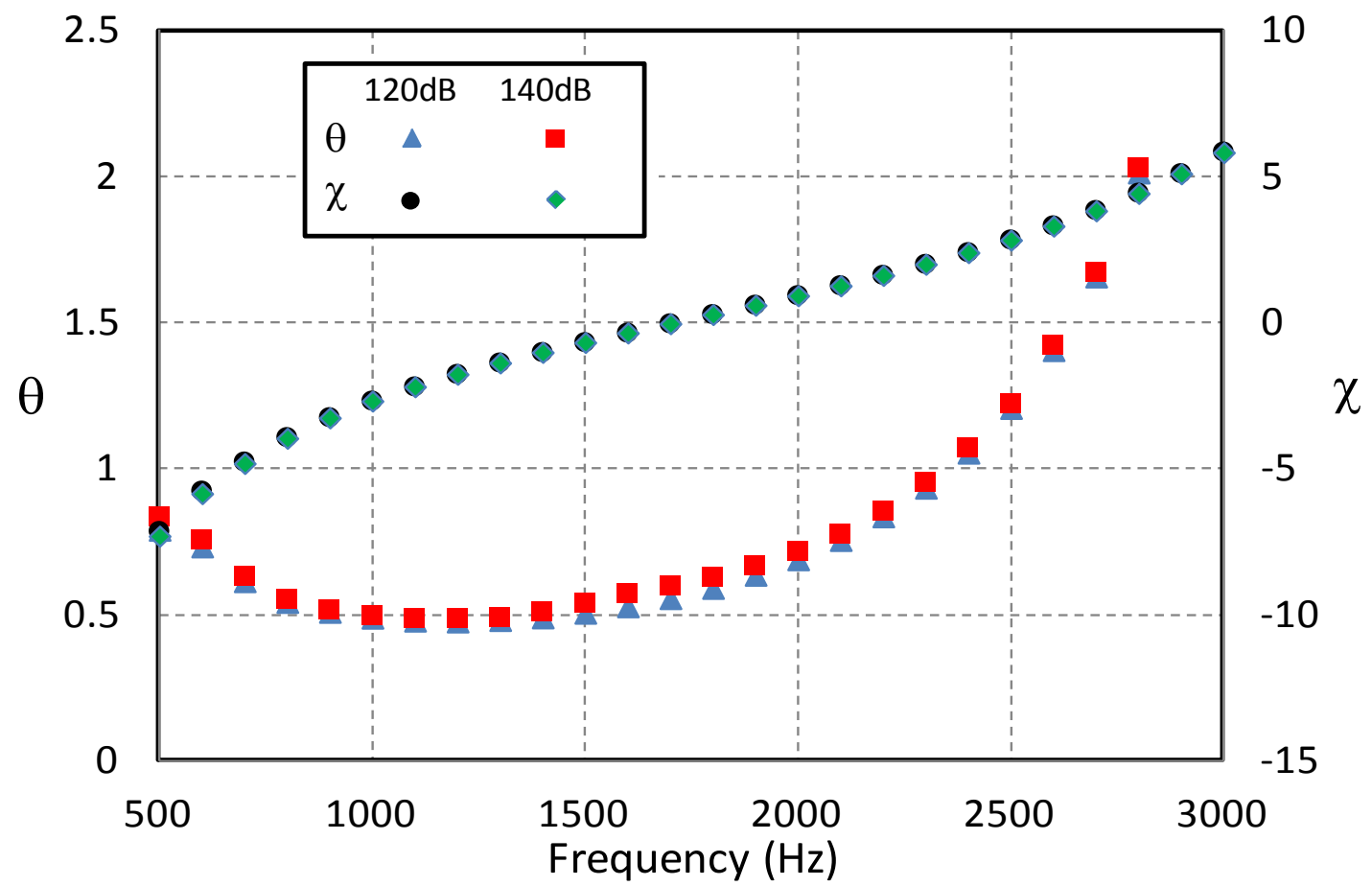

Figure 19. Measured normalized impedance $(\zeta=\theta+i \chi)$ spectra for Liner S1 (constant depth, square, straight channel), SPL $=120 \mathrm{~dB}$ and $140 \mathrm{~dB}$. 


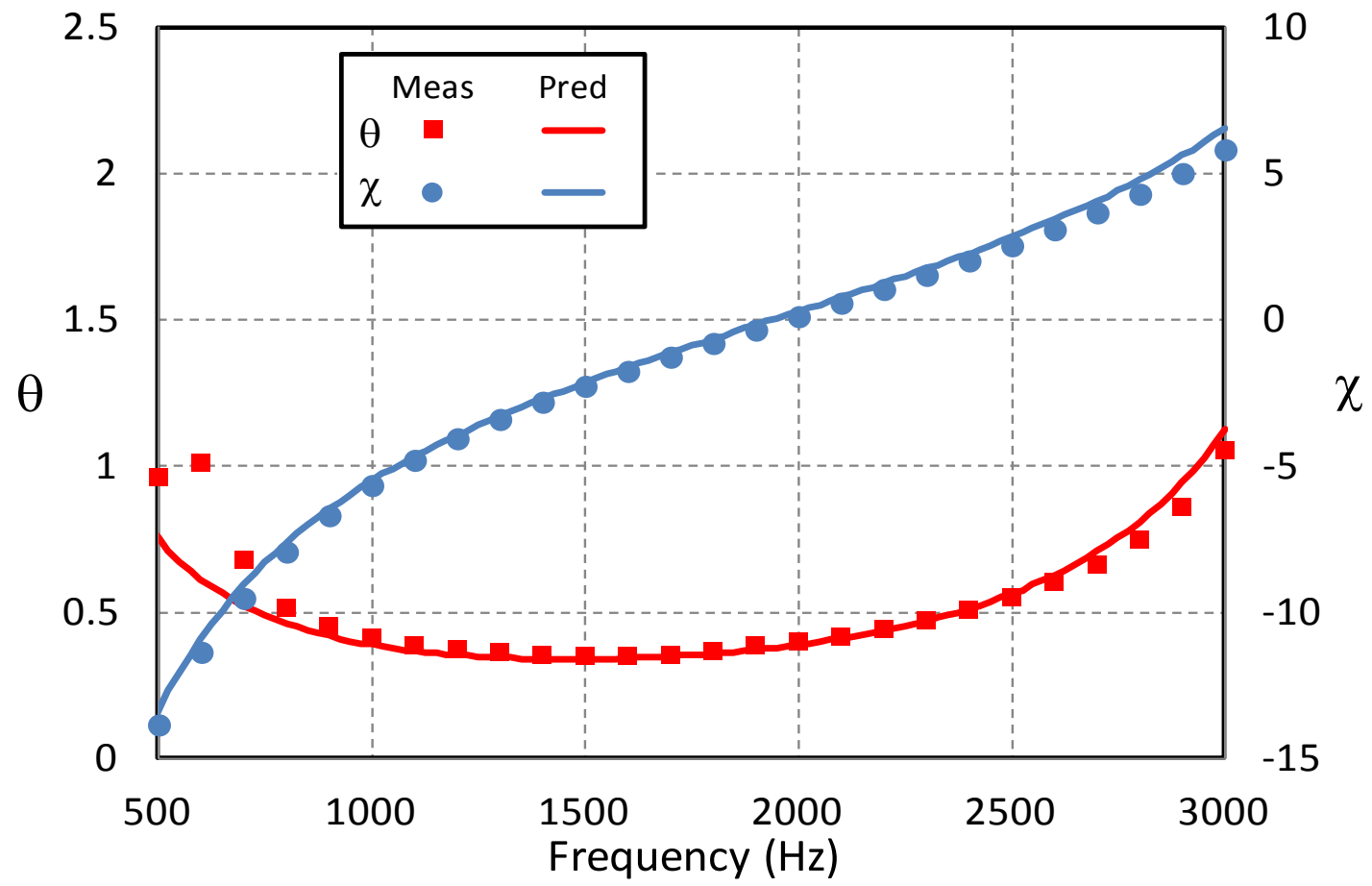

Figure 20. Measured and predicted normalized impedance $(\zeta=\theta+i \chi)$ spectra for Liner C1 (constant depth, round, straight channel).

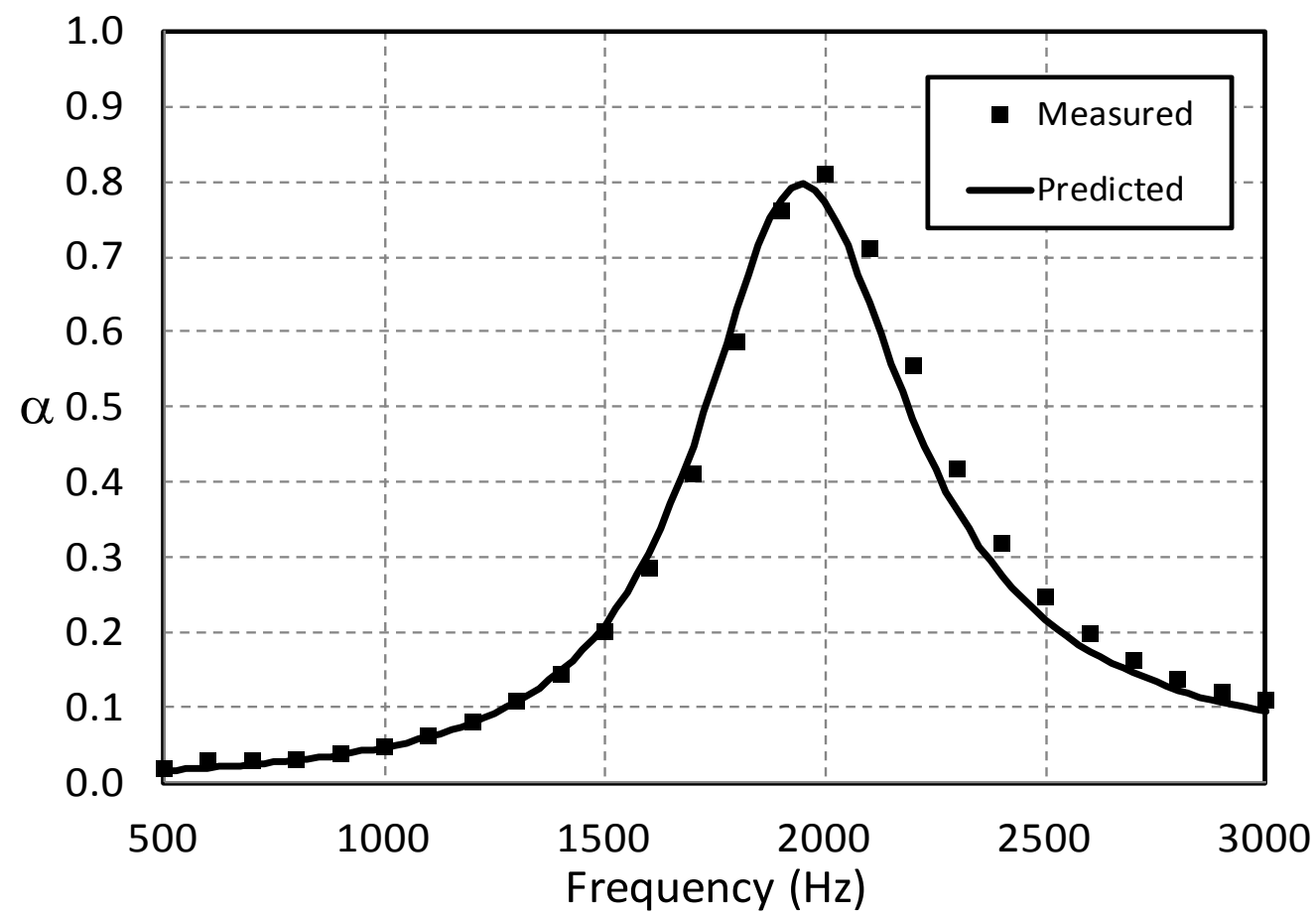

Figure 21. Measured and predicted absorption coefficient $(\alpha)$ spectra for Liner C1 (constant depth, round, straight channel). 


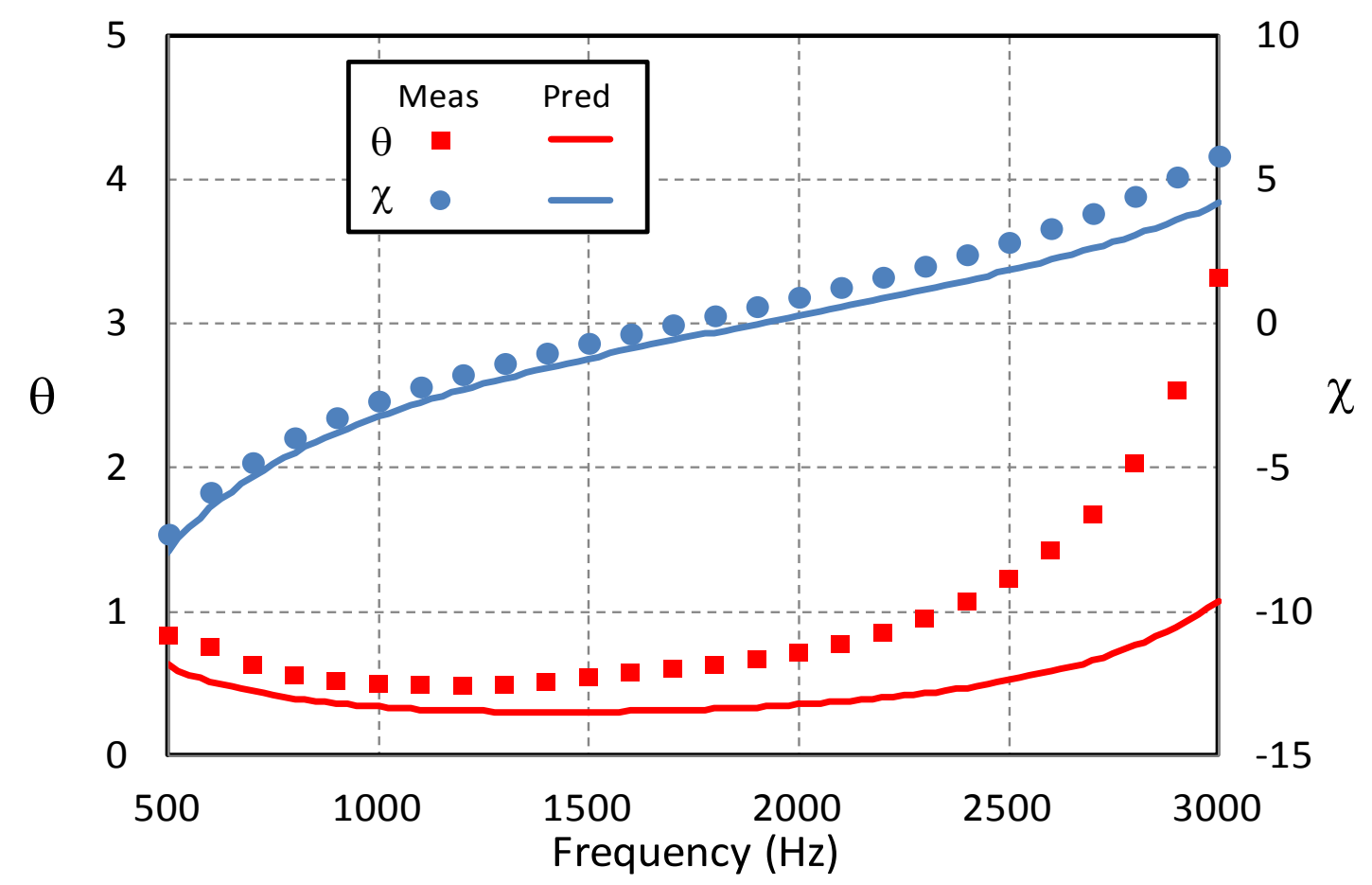

Figure 22. Measured and predicted normalized impedance $(\zeta=\theta+i \chi)$ spectra for Liner S1 (constant depth, square, straight channel).

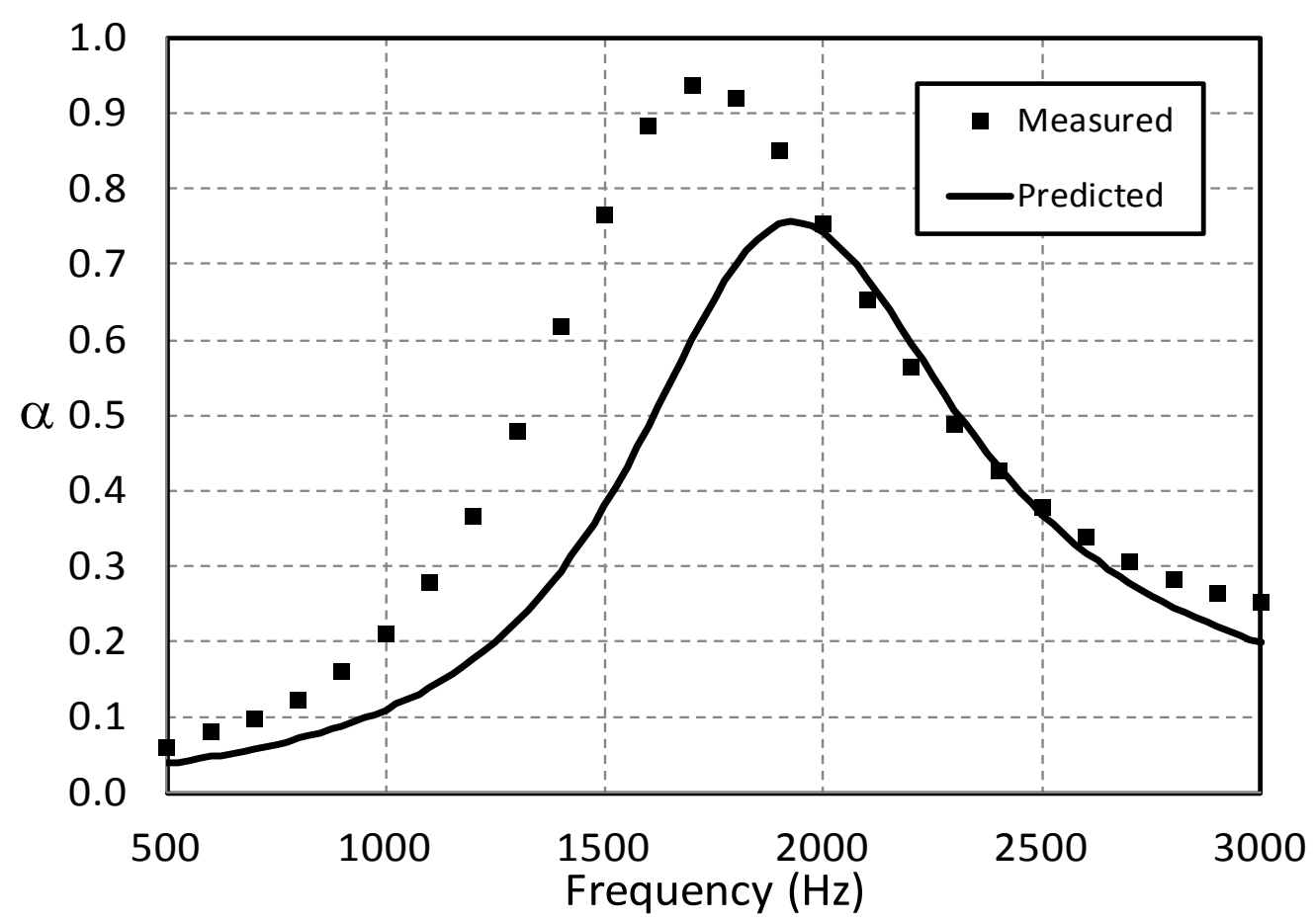

Figure 23. Measured and predicted absorption coefficient $(\alpha)$ spectra for Liner S1 (constant depth, square, straight channel). 


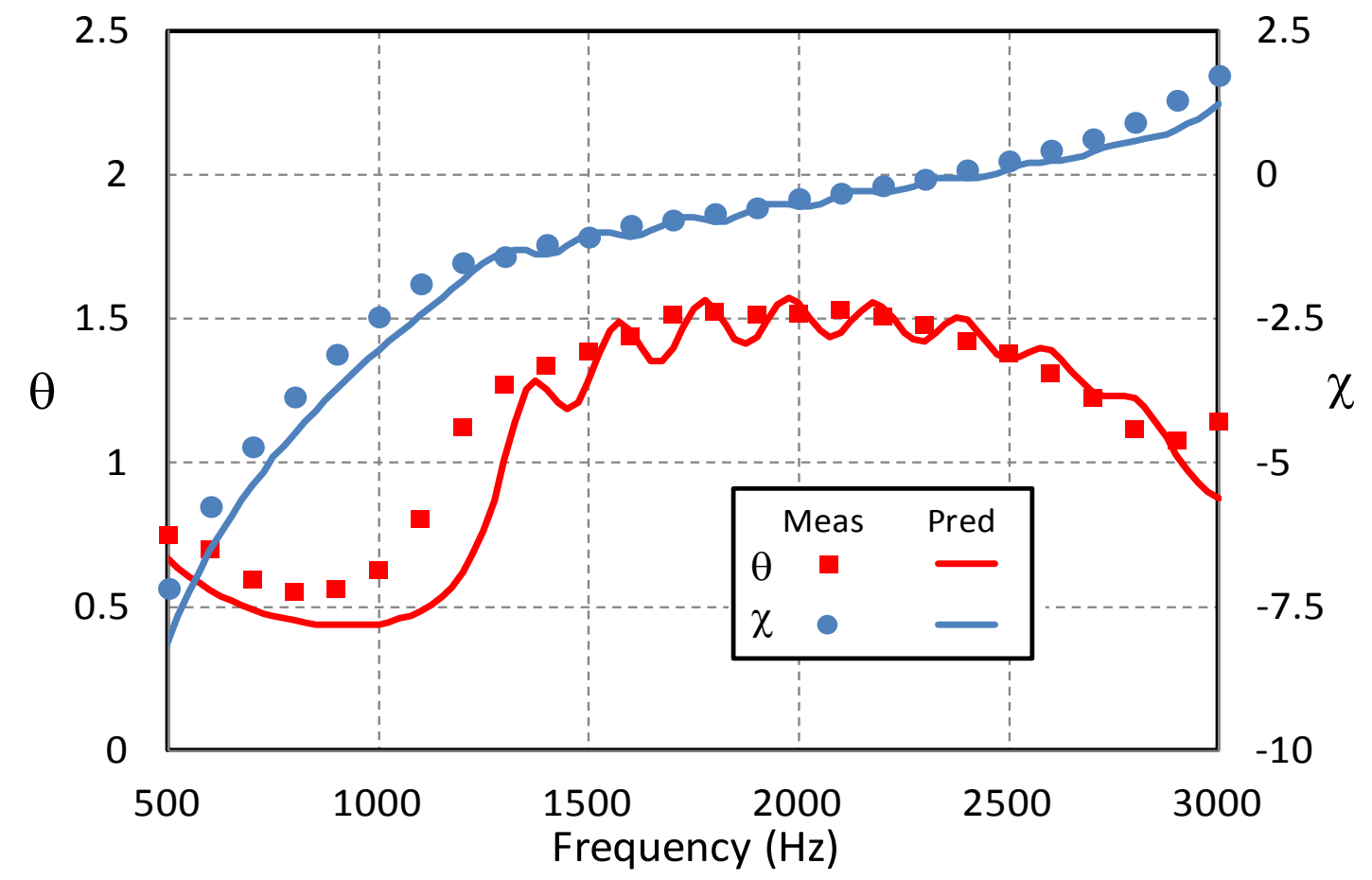

Figure 24. Measured and predicted normalized impedance $(\zeta=\theta+i \chi)$ spectra for Liner S2 (variable depth, square, straight channel).

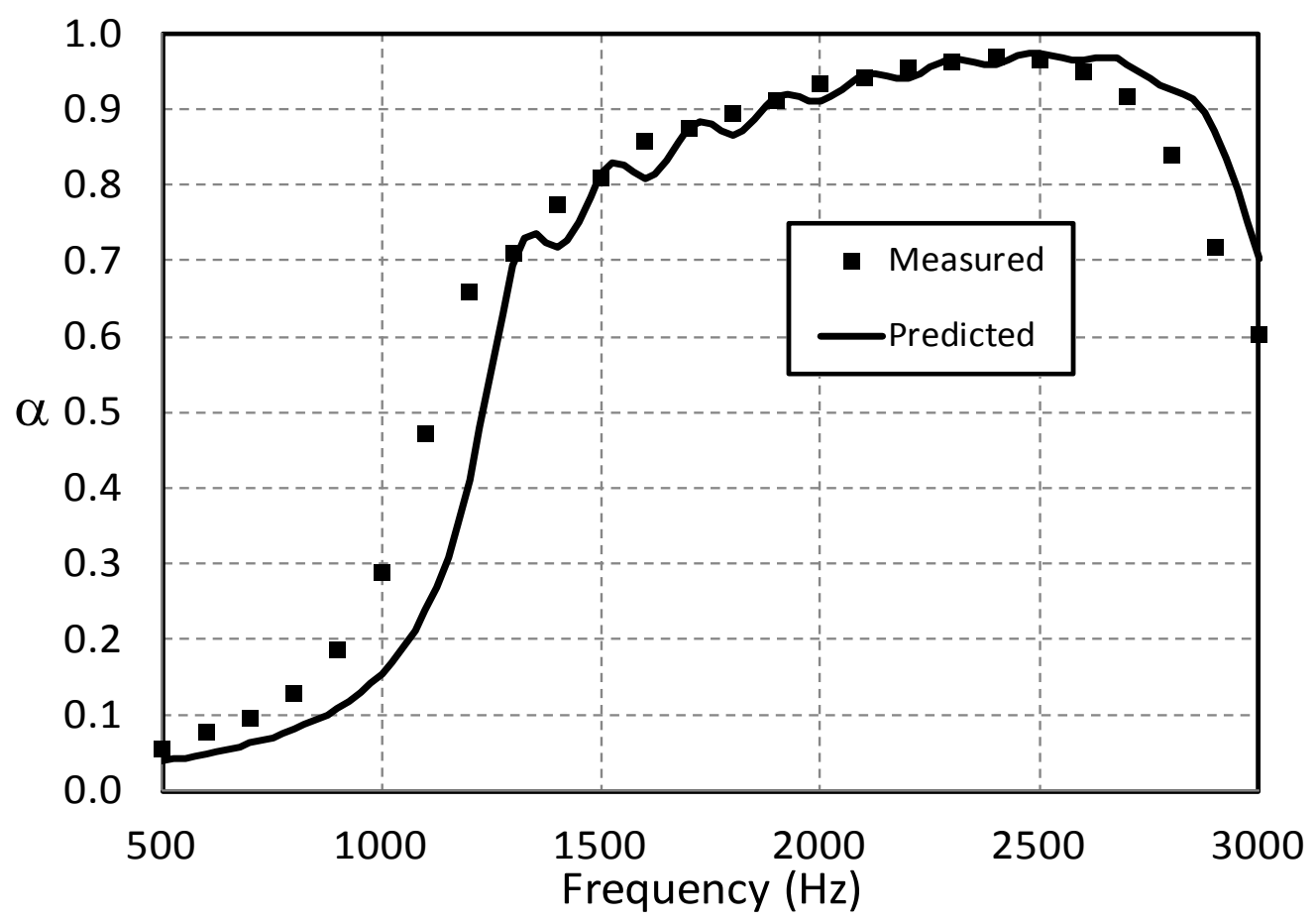

Figure 25. Measured and predicted absorption coefficient $(\alpha)$ spectra for Liner S2 (variable depth, square, straight channel). 


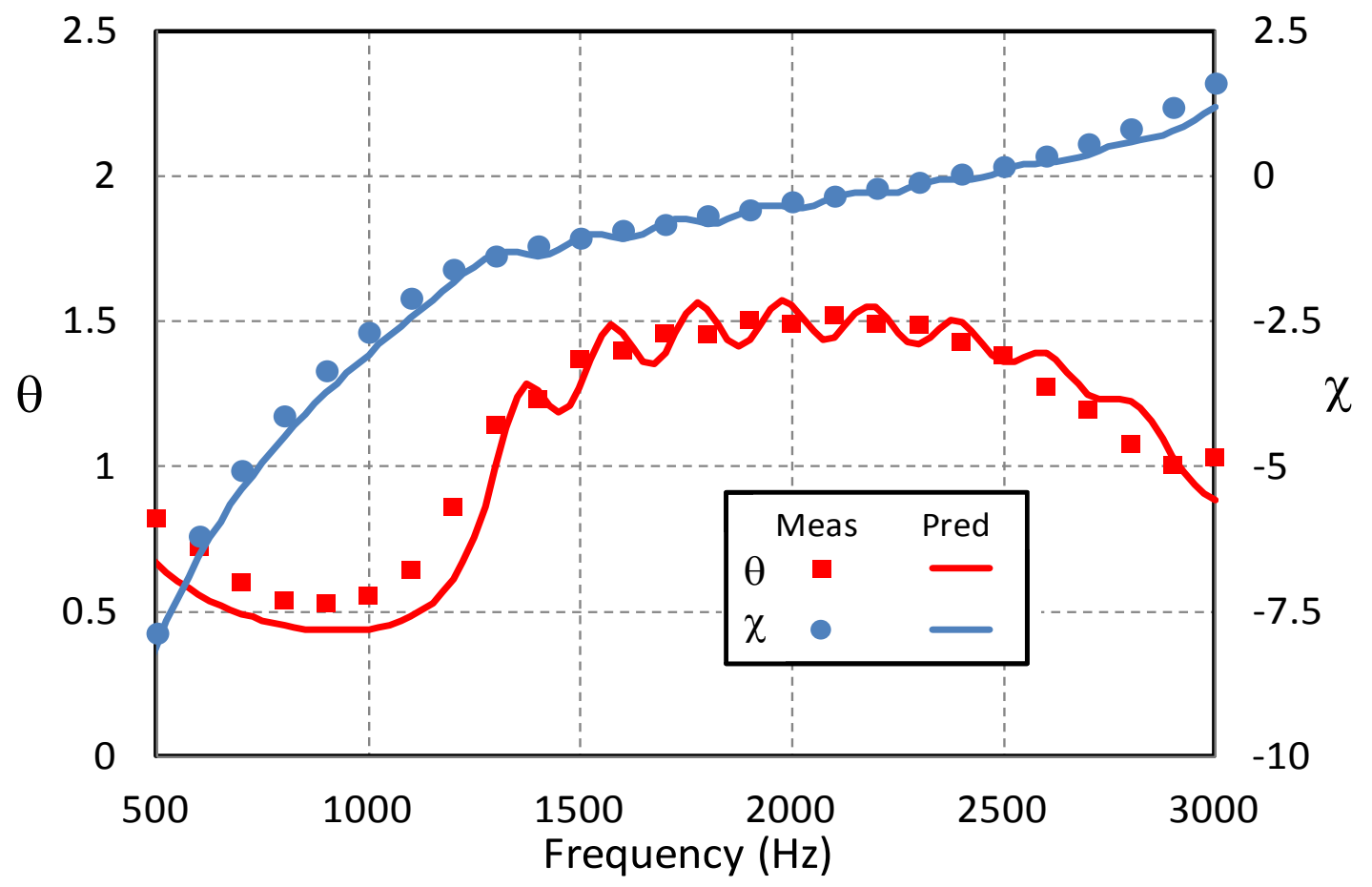

Figure 26. Measured and predicted normalized impedance $(\zeta=\theta+i \chi)$ spectra for Liner S3 (variable depth, square, bent channel).

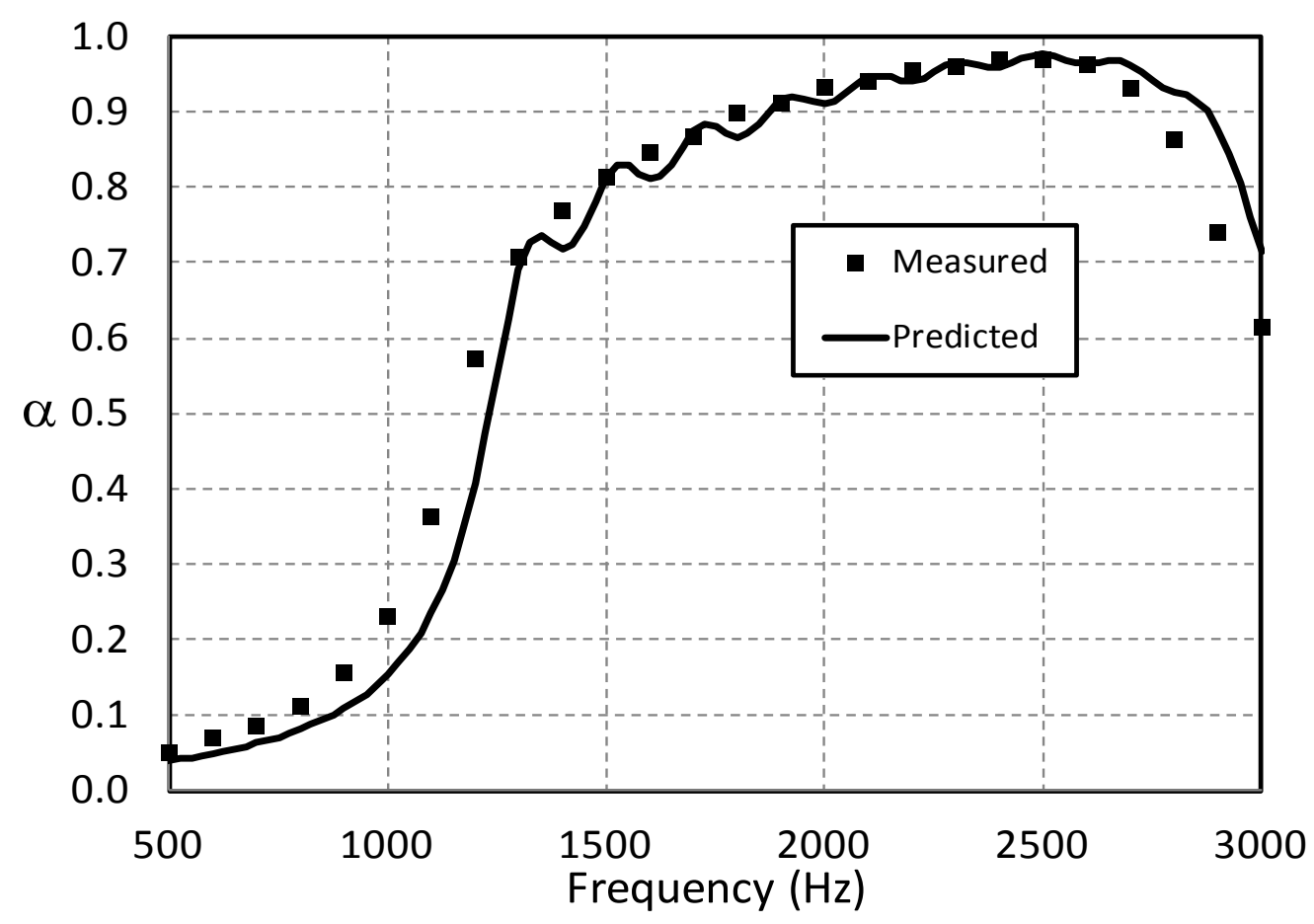

Figure 27. Measured and predicted absorption coefficient $(\alpha)$ spectra for Liner S3 (variable depth, square, bent channel). 